\title{
Food and Mood Survey
}

Start of Block: Consent Form (not included here)

Thank you for deciding to participate in this study, we greatly appreciate it!

In this first part of the survey, you will be asked about your mental wellbeing. Please note that some of the questions ask about a certain time frame (e.g. in the last 2 weeks or during the past month), others ask if you have ever experienced certain things. Please make sure you read the questions carefully.

Remember that answering these questions is voluntary. If you choose not to answer a certain question, please check the box "prefer not to say" in order to move on to the next question.

Some of the questions might make you sad or make you remember difficult times in your life. Should you experience any stress as a result from this survey, please do not hesitate to contact either one of the study staff or UBC Counselling Services located in Room 1040 in Brock Hall, 1874 East Mall or Empower Me1-844-741-6389 (toll-free). For crisis or after-hours support services, please contact the service you are most comfortable with from this list. 
In general, would you say your quality of life is:

Excellent
Very good
Good
Fair
Poor
Prefer not to say

In general, how would you rate your satisfaction with your social activities and relationships?

Excellent
Very good
Good
Fair
Poor
Prefer not to say


I am able to balance my academic time (in class, study time, etc.) and non-academic time (work, exercise, socializing, care for dependents etc.)

Strongly agree

Agree

Somewhat agree

Somewhat disagree

Disagree

Strongly disagree

Prefer not to say

I am confident that I will succeed in all of my courses

Strongly agree

Agree

Somewhat agree

Somewhat disagree

Disagree

Strongly disagree

Prefer not to say 
Within the last 12 months, how would you rate the overall level of stress you have experienced?
No stress
Less than average stress
Average stress
More than average stress
Tremendous stress
Prefer not to say

Over the last 2 weeks, how often have you been bothered by any of the following problems?

\begin{tabular}{|c|c|c|c|c|c|}
\hline & Not at all & Several days & $\begin{array}{l}\text { More than half } \\
\text { the days }\end{array}$ & $\begin{array}{c}\text { Nearly every } \\
\text { day }\end{array}$ & $\begin{array}{c}\text { Prefer not to } \\
\text { say }\end{array}$ \\
\hline $\begin{array}{l}\text { Little interest } \\
\text { or pleasure in } \\
\text { doing things }\end{array}$ & & & & & \\
\hline $\begin{array}{l}\text { Feeling down, } \\
\text { depressed, or } \\
\text { hopeless }\end{array}$ & & & & & D \\
\hline $\begin{array}{l}\text { Trouble falling } \\
\text { or staying } \\
\text { asleep, or } \\
\text { sleeping too } \\
\text { much }\end{array}$ & & & & & \\
\hline $\begin{array}{c}\text { Feeling tired or } \\
\text { having little } \\
\text { energy }\end{array}$ & & & & & \\
\hline $\begin{array}{l}\text { Poor appetite } \\
\text { or overeating }\end{array}$ & & & & & \\
\hline $\begin{array}{l}\text { Feeling bad } \\
\text { about yourself } \\
\text { or that you are } \\
\text { a failure or } \\
\text { have let } \\
\text { yourself or } \\
\text { your family } \\
\text { down }\end{array}$ & 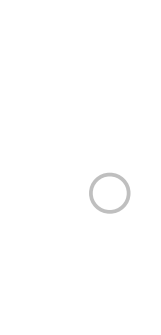 & $\cap$ & & & \\
\hline
\end{tabular}




Trouble
concentrating
on things, such
as reading the
newspaper or
watching
television

Moving or
speaking so
slowly that
other people
could have
noticed. Or the
opposite -
being so
fidgety or
restless that
you have been
moving around
a lot more than
usual
Thoughts that
you would be
better off dead
or of hurting
yourself

Thinking about the problems you checked off in the previous question, how difficult have these problems made it for you to do your work, take care of things at home, or get along with other people?

Not difficult at all

Somewhat difficult

Very difficult

Extremely difficult

Prefer not to say 
Over the last 2 weeks, how often have you been bothered by the following problems?

\begin{tabular}{c|c|cc} 
Feeling & Not at all \\
nervous, \\
anxious, or \\
on edge \\
$\begin{array}{c}\text { Not being } \\
\text { able to stop } \\
\text { or control } \\
\text { worrying }\end{array}$ \\
Worrying too \\
much about \\
different \\
things \\
Trouble \\
relaxing
\end{tabular}


Have you ever been diagnosed or treated by a professional for any of the following? Check all that apply.

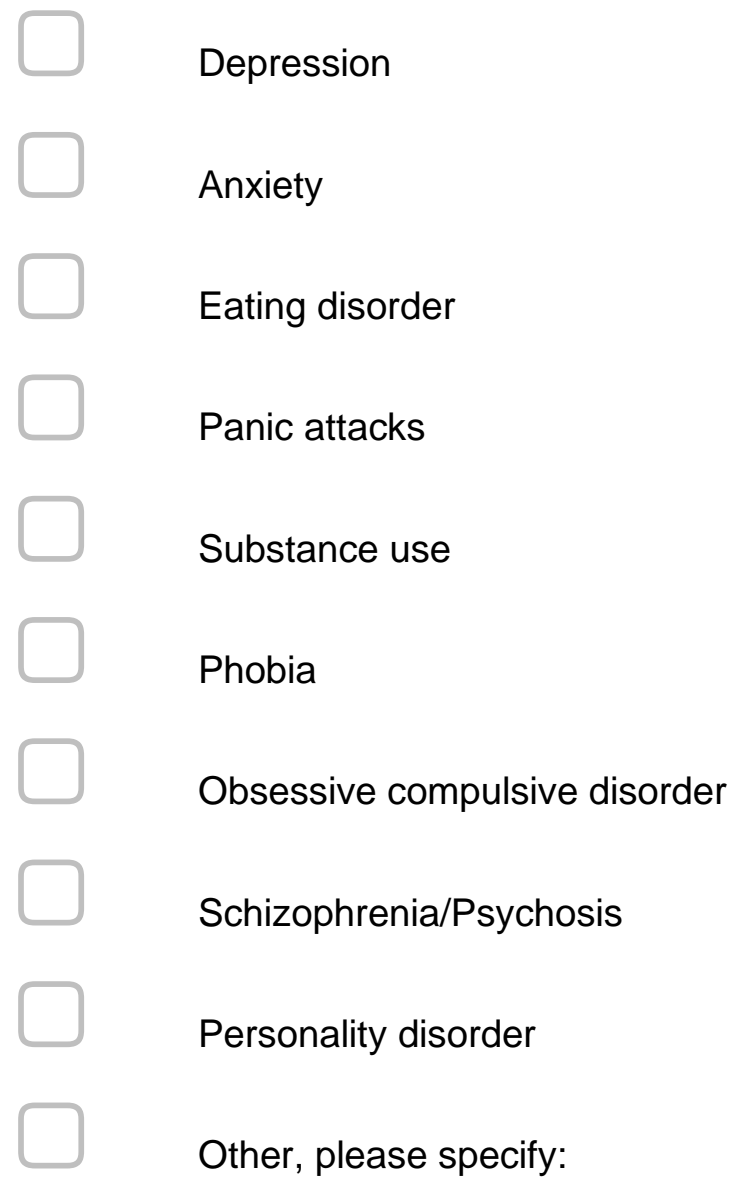
I have never been diagnosed with any of the above mentioned disorders $\square$ Prefer not to say

How old were you when you were first diagnosed with \{automatic entry from previous questions\}? 
Have you ever struggled with any of the following mental health issues that were not diagnosed by a professional? Check all that apply.

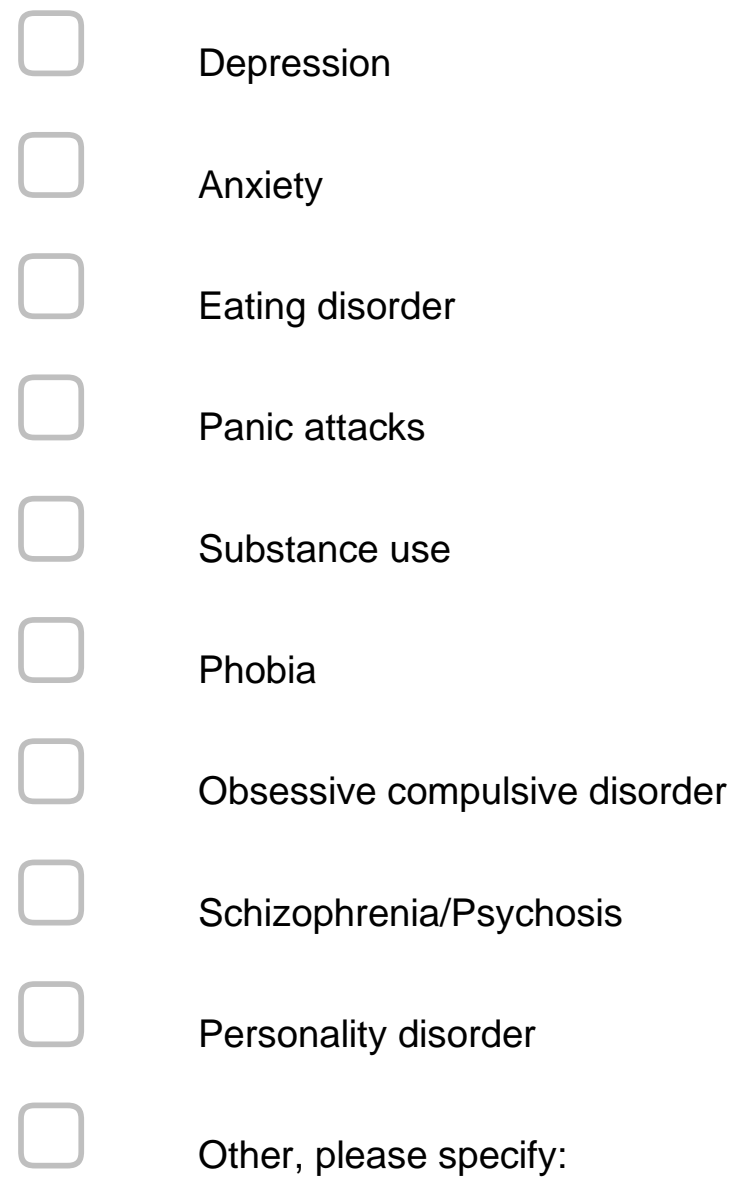

I have never struggled with any of the above mentioned issues

Prefer not to say

How old were you when you first struggled with \{automatic entry from previous question\}? 
Does anyone in your family have a history of mental illness? Check all that apply.

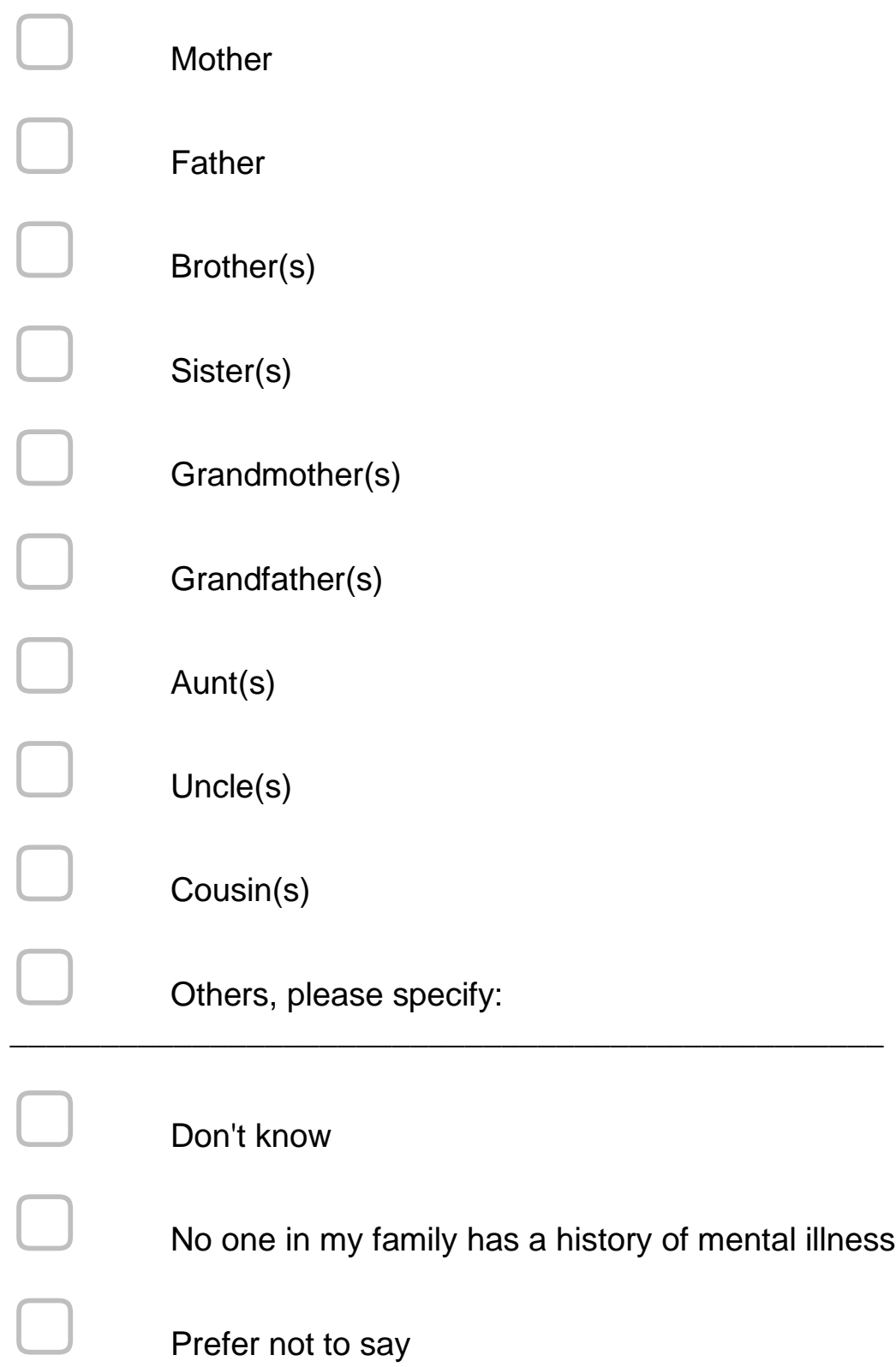


Are you satisfied with your eating patterns?
Yes
No
Prefer not to say

Do you ever eat in secret?
Yes
No
Prefer not to say

Does your weight affect the way you feel about yourself?
Yes
No
Prefer not to say

Have any family members of your family suffered with an eating disorder?
Yes
No
Don't know
Prefer not to say 
How do you describe your weight?

Very underweight

Slightly underweight

About the right weight

Slightly overweight

Very overweight

Prefer not to say

How satisfied are you with your current weight?

Not satisfied at all

Slightly unsatisfied

Somewhat satisfied

Very satisfied

Extremely satisfied

Prefer not to say 
Are you trying to do any of the following about your weight?

I am not trying to do anything about my weight

Stay the same weight

Lose weight

Gain weight

Prefer not to say

Thinking back over the past $\mathbf{3 0}$ days, how many cigarettes (the kind that come in a pack or rollyour-own) did you smoke in a normal week?
None
$1-5 /$ week
6-15/week
about a pack/week
more than a pack/week
Prefer not to say 
Thinking back over the past $\mathbf{3 0}$ days, how often did you consume marijuana in a normal week?

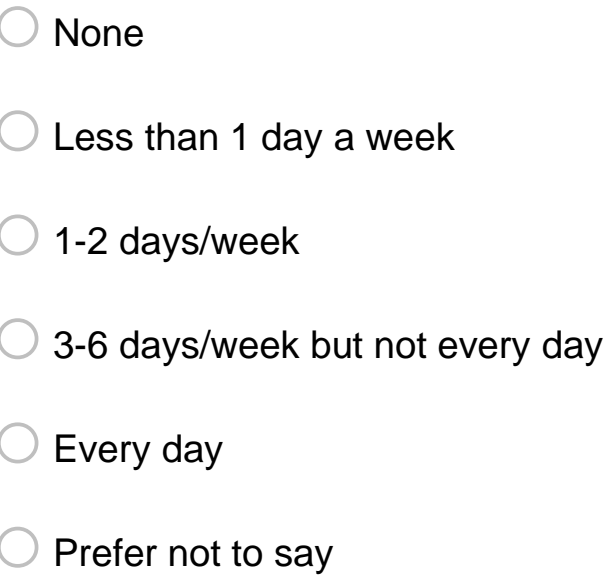

Thinking back over the past $\mathbf{3 0}$ days, how often did you consume alcohol in a normal week?

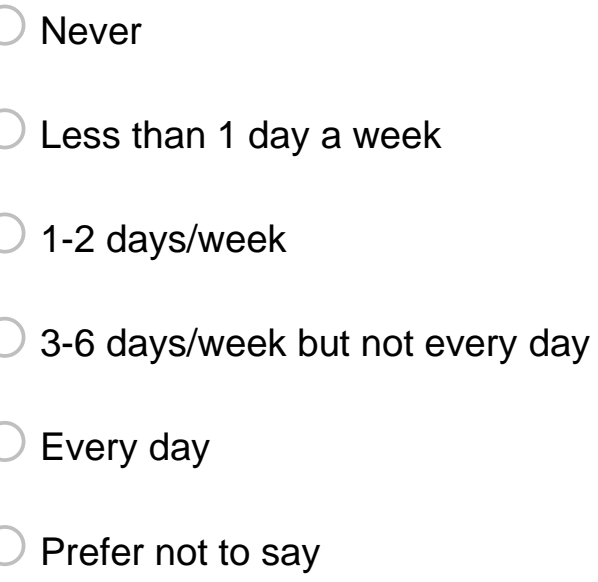


In the past year, have any of these events occurred in your life? Check all that apply.

Death of a close family member

Death of a close friend

Divorce between parents

Serious legal problems

Major personal injury or illness

Responsibilities for others such as children/spouse

Threat to major source of income

Difficulty with roommate(s)

Change in health of a family member

Pregnancy

Sexual problems

Serious disagreements with parents

Change in lifestyle for financial reasons

Difficulty in identifying a major

Serious argument with close family member

Problems with a girlfriend or boyfriend

Having to repeat a course

Increased workload at school 
Outstanding personal achievement

First semester in college

Change in living conditions

Serious disagreements with an instructor

Lower grades than expected

Change in sleeping habits

Change in social habits

Change in eating habits

Chronic car problems

Change in number of family get togethers

Too many missed classes

Change in plans for a major

Dropped more than one class

Minor traffic violations

Prefer not to say 
In general, how would you rate your physical health?
Excellent
Very good
Good
Fair
Poor
Prefer not to say

On how many of the past $\mathbf{7}$ days did you participate in vigorous exercise for at least 20 minutes or moderate exercise for at least 30 minutes? Vigorous exercise are activities that take hard physical effort and make you breathe much harder than normal. Moderate exercise are activities that take moderate physical effort and make you breathe somewhat harder than normal.

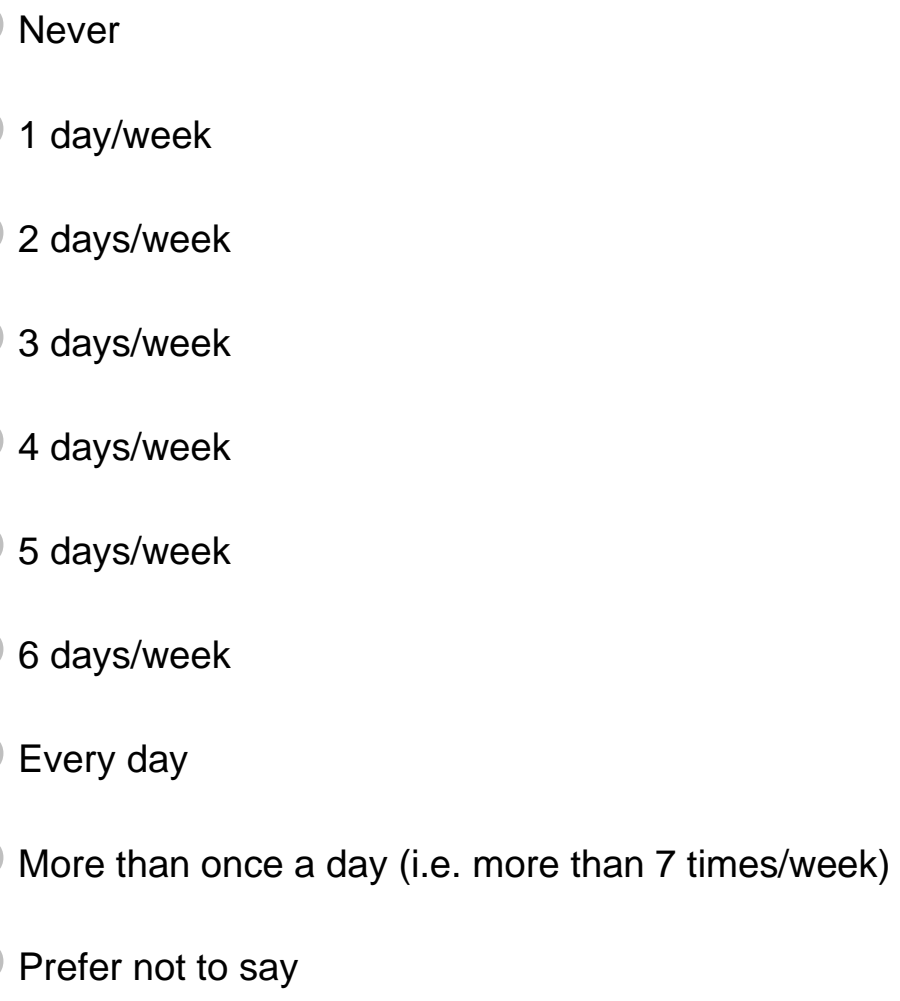


On how many of the past 7 days did you get enough sleep so that you felt rested when you woke up in the morning?
Never
1 day/week
2 days/week
3 days/week
4 days/week
5 days/week
6 days/week
Every day
Prefer not to say

Do you currently take any medication for mental health issues?
Yes
No
Prefer not to say

Please tell us more about the medication you take for mental health issues:

Name of the medication

Dosage you take (e.g. one capsule of $20 \mathrm{mg} /$ day) 
Great, part one is done! Now we will move on to the second part of the survey.

The following questions will ask about your diet, food allergies, and more. Please answer every question, failure to do so will lead your responses to be unusable for this study. If you are unsure about the answer, try to give your best estimate.

The questions are about foods you ate and drinks you drank during the past month, that is, the past 30 days. When answering, please include meals and snacks at home, at work or school, in restaurants and anyplace else.

During the past month, how often did you eat hot or cold cereals? Check one.
Never
$1 x /$ month
$2-3 x /$ month
$1 \times /$ week
$2 x /$ week
3-4x/week
$5-6 x /$ week
1x/day
2 or more times/day

During the past month, what kind of cereal did you usually eat? 
If there was another kind of cereal that you usually ate during the past month, what kind was it?

During the past month, how often did you have any dairy milk (either to drink or on cereal)? Include regular milks, chocolate or flavored milks, lactose-free milk, buttermilk. Please do not include small amounts of milk in coffee or tea. Do not include soy milk or other non-dairy milks such as almond milk. Check one.
Never
$1 \mathrm{x} / \mathrm{month}$
$2-3 x /$ month
$1 \mathrm{x} /$ week
$2 x /$ week
3-4x/week
5-6x/week
$1 \mathrm{x} /$ day
$2-3 x / d a y$
$4-5 x /$ day
6 or more times/day 
During the past month, what kind of dairy milk did you usually drink? Check one.

Whole or regular milk

$2 \%$ fat or reduced-fat milk

$1 \%$ or $0.5 \%$ or low-fat milk

Fat-free, skim or non-fat milk

Other kind of milk, please specify:

During the past month, how often did you have any non-dairy milk such as soy milk, almond milk, rice milk etc. (either to drink or on cereal)? Please do not include small amounts of milk in coffee or tea. Check one.
Never
$1 \mathrm{x} / \mathrm{month}$
$2-3 x /$ month
$1 \mathrm{x} /$ week
$2 x /$ week
$3-4 x /$ week
$5-6 x /$ week
$1 \times /$ day
2 or more times/day 
During the past month, how often did you drink regular soda or pop that contains sugar? Do not include diet soda. Check one.
Never
$1 x /$ month
2-3x/month
$1 x /$ week
2x/week
$3-4 x /$ week
5-6x/week
$1 x /$ day
2-3x/day
$4-5 x / d a y$
6 or more times/day 
During the past month, how often did you drink $100 \%$ pure fruit juices such as orange, mango, apple, grape and pineapple juice? Do not include fruit-flavored drinks with added sugar or fruit juice you made at home and added sugar to. Check one.
Never
$1 \mathrm{x} /$ month
$2-3 x /$ month
$1 \mathrm{x} /$ week
2x/week
$3-4 x /$ week
$5-6 x /$ week
1x/day
2-3x/day
4-5x/day
6 or more times/day

During the past month, how often did you drink coffee or tea that had sugar or honey added to it? Include coffee and tea you sweetened yourself and pre-sweetened tea and coffee drinks 
such as Arizona Iced Tea or Frappuccino. Do not include artificially sweetened coffee or diet tea. Check one.
Never
$1 x /$ month
$2-3 x /$ month
$1 \mathrm{x} /$ week
$2 x /$ week
$3-4 x /$ week
$5-6 x /$ week
$1 \times /$ day
2-3x/day
4-5x/day
6 or more time

During the past month, how often did you drink sweetened fruit drinks, sports or energy drinks, such as Kool-Aid, lemonade, Hi-C, cranberry drink, Gatorade, Red Bull or Vitamin Water? 
Include fruit juices you made at home and added sugar to. Do not include diet drinks or artificially sweetened drinks. Check one.
Never
$1 x /$ month
2-3x/month
$1 x /$ week
2x/week
$3-4 x /$ week
5-6x/week
$1 \mathrm{x} /$ day
2-3x/day
4-5x/day
6 or more times/day 
During the past month, how often did you eat fruit? Include fresh, frozen or canned fruit. Do not include juices. Check one.
Never
$1 \mathrm{x} /$ month
$2-3 x /$ month
$1 \mathrm{x} /$ week
$2 x /$ week
$3-4 x /$ week
5-6x/week
$1 \times /$ day
2 or more times/day

During the past month, how often did you eat a green leafy or lettuce salad, with or without other vegetables? Check one.
Never
$1 x /$ month
$2-3 x /$ month
$1 \mathrm{x} /$ week
2x/week
$3-4 x /$ week
5-6x/week
1x/day
2 or more times/day 
During the past month, how often did you eat any kind of fried potatoes, including French fries, home fries, or hash brown potatoes? Check one.
Never
$1 \mathrm{x} /$ month
$2-3 x /$ month
$1 \mathrm{x} /$ week
2x/week
$3-4 x /$ week
5-6x/week
$1 x /$ day
2 or more times/day 
During the past month, how often did you eat any other kind of potatoes, such as baked, boiled, mashed potatoes, sweet potatoes, or potato salad? Check one.

Never
$1 x /$ month
$2-3 x /$ month
1 x/week
$2 x /$ week
$3-4 x /$ week
$5-6 x /$ week
$1 x /$ day
2 or more times/day


During the past month, how often did you eat cooked dried beans or canned beans and legumes such as baked beans, pinto beans, kidney beans, lima beans, lentils, soybeans, chickpeas, or refried beans? Do not include green beans. Check one.
Never
$1 \mathrm{x} /$ month
$2-3 x /$ month
$1 x /$ week
$2 x /$ week
$3-4 x /$ week
5-6x/week
1x/day
2 or more times/day 
During the past month, how often did you eat brown rice or other cooked whole grains, such as quinoa, barley, bulgur, cracked wheat, or millet? Do not include white rice. Check one.
Never
$1 \mathrm{x} /$ month
$2-3 x /$ month
$1 \mathrm{x} /$ week
$2 x /$ week
$3-4 x /$ week
$5-6 x /$ week
1x/day
2 or more times/day

During the past month, not including what you just told us about (green salads, potatoes, cooked dried beans etc.) how often did you eat other vegetables? Check one.
Never
$1 x /$ month
$2-3 x /$ month
1x/week
$2 x /$ week
3-4x/week
5-6x/week
1x/day
2 or more times/day 
During the past month, how often did you eat nuts and seeds such as peanuts, cashews, walnuts, pecans, sunflower seeds or pumpkin seeds? Check one.
Never
$1 \mathrm{x} /$ month
$2-3 x /$ month
$1 \mathrm{x} /$ week
$2 x /$ week
$3-4 x /$ week
$5-6 x /$ week
$1 \times /$ day
2 or more times/day 
During the past month, how often did you eat pizza? Include frozen pizza, fast food pizza, and homemade pizza. Check one.
Never
$1 \mathrm{x} /$ month
$2-3 x /$ month
$1 \mathrm{x} /$ week
$2 x /$ week
$3-4 x /$ week
$5-6 x /$ week
$1 \mathrm{x} /$ day
2 or more times/day

During the past month, how often did you eat tomato sauces such as with spaghetti or noodles or mixed into foods such as lasagna? Do not include tomato sauce on pizza. Check one.
Never
$1 \mathrm{x} /$ month
$2-3 x /$ month
$1 \mathrm{x} /$ week
2x/week
3-4x/week
5-6x/week
1x/day
2 or more times/day 
During the past month, how often did you eat any kind of cheese? Include cheese as a snack, cheese on burgers, sandwiches, and cheese in foods such as lasagna quesadillas or casseroles. Do not include cheese on pizza. Check one.
Never
$1 \mathrm{x} /$ month
$2-3 x /$ month
$1 \mathrm{x} /$ week
2x/week
$3-4 x /$ week
5-6x/week
$1 \times /$ day
2 or more times/day 
During the past month, how often did you eat yogurt, Greek yogurt or other dairy products? Do not include fluid dairy milk or cheese. Check one.

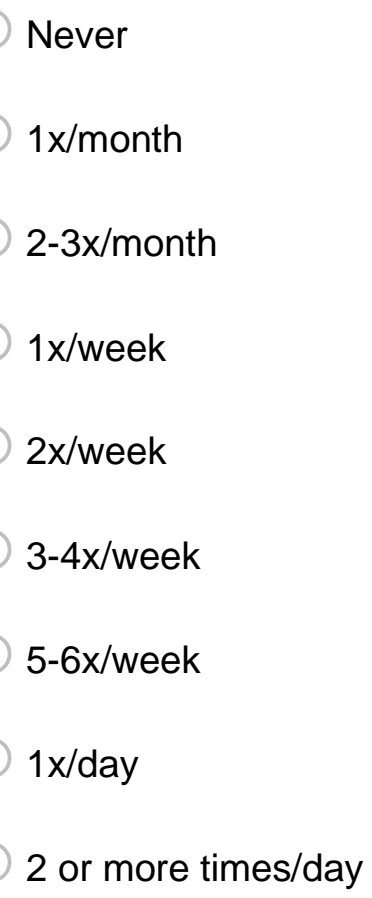

During the past month, how often did you eat red meat, such as beef, pork ham, or sausage? Include red meat you had in sandwiches, lasagna, stew, and other mixtures. Red meats may 
also include veal, lamb, and any lunch meats made with these meats. Do not include chicken, turkey, fish or seafood. Check one.

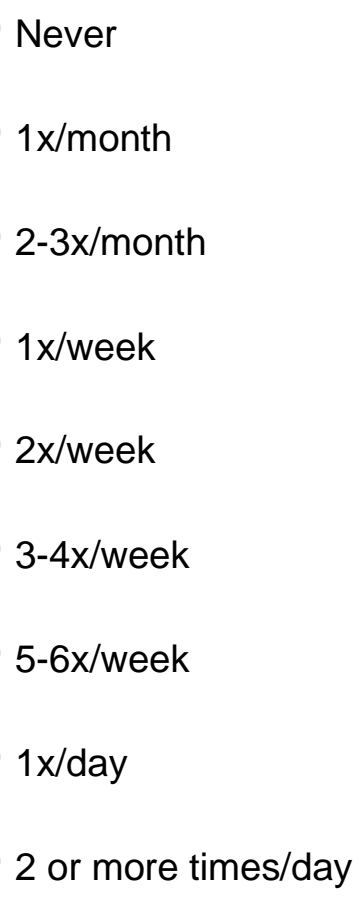

During the past month, how often did you eat any processed meat, such as bacon, lunch meats, or hot dogs? Include processed meats you had in sandwiches, soups, pizza, casseroles, and other mixtures. Processed meats are those preserved by smoking, curing or salting or by 
the addition of preservatives. Examples are: ham, bacon, pastrami, salami, sausages, bratwursts, frankfurters, hot dogs or spam. Check one.
Never
$1 \mathrm{x} /$ month
$2-3 x /$ month
$1 x /$ week
$2 x /$ week
$3-4 x /$ week
$5-6 x /$ week
1x/day
2 or more times/day

During the past month, how often did you eat poultry such as chicken or turkey? Include poultry you had in sandwiches, lasagna, stew, and other mixtures. Check one.
Never
$1 x /$ month
$2-3 x /$ month
$1 x /$ week
$2 x /$ week
$3-4 x /$ week
$5-6 x /$ week
$1 x /$ day
2 or more times/day 
During the past month, how often did you eat fish or seafood? Check one.
Never
$1 \mathrm{x} /$ month
$2-3 x /$ month
1x/week
2x/week
3-4x/week
5-6x/week
1x/day
2 or more times/day

During the past month, how often did you eat vegetarian meat alternatives and soy products such as tofu, vegetarian sausage, vegetarian sandwich meats etc.? Check one.
Never
$1 x /$ month
2-3x/month
$1 \mathrm{x} /$ week
2x/week
3-4x/week
5-6x/week
$1 x / d a y$
2 or more times/day 
During the past month, how often did you eat whole grain bread including toast, rolls, and in sandwiches? Whole grain breads include whole wheat, rye, oatmeal and pumpernickel. Do not include white bread or multigrain bread. Check one.

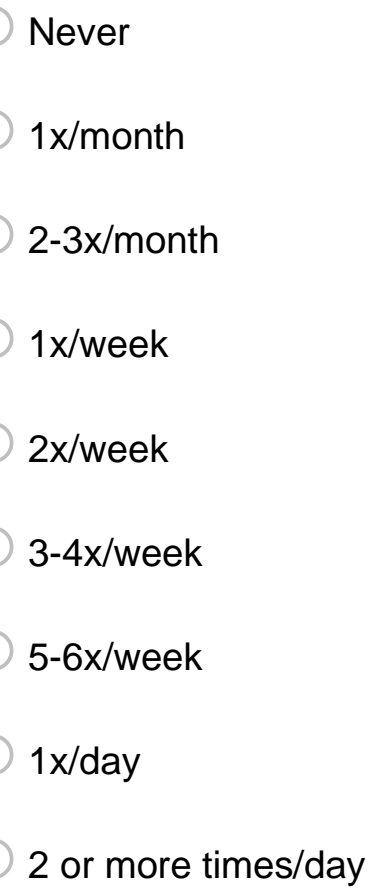


During the past month, how often did you eat chocolate or any other types of candy? Do not include sugar-free candy. Check one.
Never
$1 \mathrm{x} /$ month
$2-3 x /$ month
$1 \mathrm{x} /$ week
2x/week
$3-4 x /$ week
5-6x/week
1x/day
2 or more times/day

During the past month, how often did you eat doughnuts, sweet rolls, danish, muffins, pan dulce, or pop tarts? Do not include sugar-free items. Check one.
Never
$1 \mathrm{x} /$ month
$2-3 x /$ month
$1 x /$ week
$2 x /$ week
$3-4 x /$ week
$5-6 x /$ week
$1 \mathrm{x} /$ day
2 or more times/day 
During the past month, how often did you eat cookies, cake, pie or brownies? Do not include sugar-free kinds. Check one.

Never
1 x/month
$2-3 x /$ month
1 x/week
$2 x /$ week
$3-4 x /$ week
$5-6 x /$ week
$1 x /$ day
2 or more times/day


During the past month, how often did you eat ice cream or other frozen desserts? Do not include sugar-free kinds. Check one.
Never
$1 \mathrm{x} /$ month
$2-3 x /$ month
$1 \mathrm{x} /$ week
$2 x /$ week
$3-4 x /$ week
$5-6 x /$ week
$1 x /$ day
2 or more times/day 
During the past month, did you take any of the following supplements? Check all that apply.

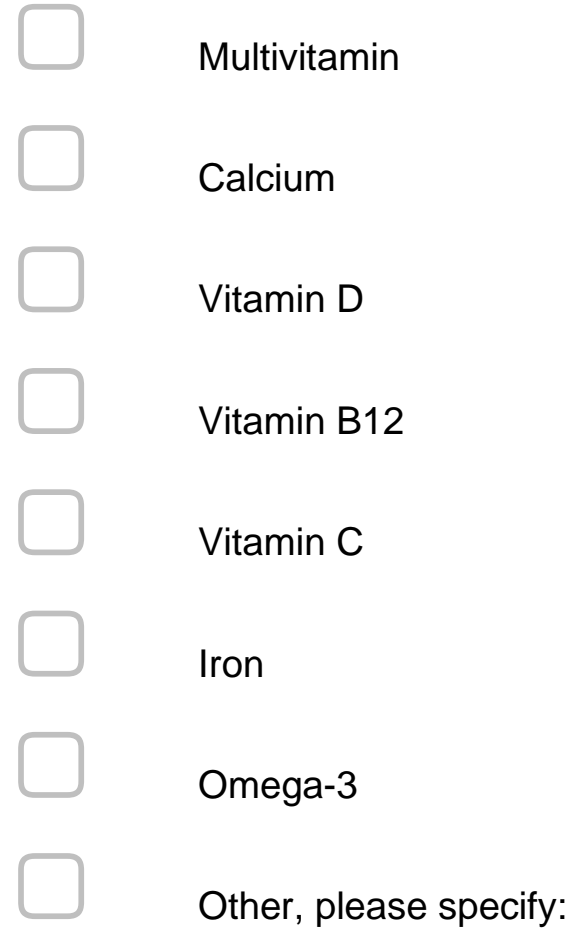

I did not take any supplements

Please specify how often (per week) and how much (units/dosage per intake) of \{automatic entry from previous question\} you took during the past month: 
Do you identify as one of the following? Check one.

Pescetarian (you eat fish, eggs, dairy, but no meat or poultry)

Vegetarian (you eat eggs and dairy, but no fish, meat or poultry)

Vegan (you don't eat any animal products)

Other, please specify:

I don't identify as any of the above

Prefer not to answer

How old were you when you first identified as \{automatic entry from previous question\}?

What is your reason to identify as \{automatic entry from previous question \}? Check the option that is your most important reason.

Weight loss

Health reasons

Ethical reasons (i.e. to reduce the suffering of animals)

Environmental reasons

Religious or cultural reasons

Other, please specify:

Prefer not to answer 
Do you have any food allergies? Check all that apply.

\section{Peanuts}

Tree nuts (such as almonds, Brazil nuts, cashews, hazelnuts, macadamias, pecans, pine nuts. pistachios, walnuts)

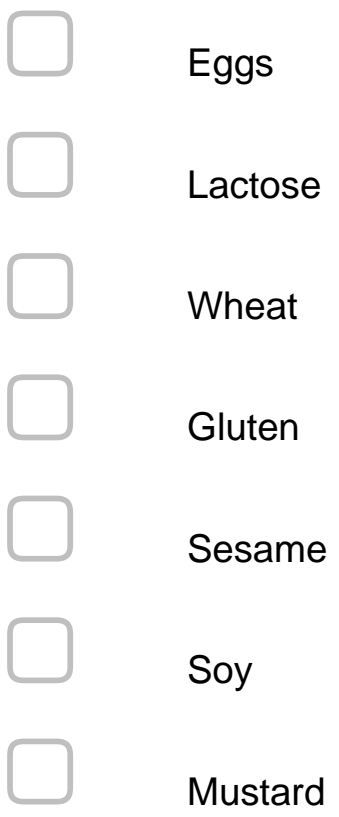

Fish

Shellfish (i.e. crustaceans and mollusks)

Other, please specify:

I have no food allergies

Prefer not to answer 
Awesome, part two is done, too!

In this final and short part of the survey, we will ask you a few questions about yourself. Remember that answering these questions is voluntary. If you choose not to answer a certain question, please check the box "prefer not to say" or leave the text box blank in order to move on to the next question.

How old are you (in years)?

Which term do you use to describe your gender identity?

Woman

Man

Trans Woman

Trans Man

Gender queer

Other, please specify:

Prefer not to say 
What term best describes your sexual orientation?
Asexual
Bisexual
Gay
Lesbian
Pan sexual
Queer
Same gender loving
Straight/heterosexual
Other, please specify:
Prefer not to say

What is your relationship status?
Not in a relationship
In a relationship but not living together
In a relationship and living together
I'm not sure
Prefer not to say

What is your height (please indicate feet and inches or centimeters)? 
What is your weight (please indicate pounds or kilograms)?

How would you describe your ethnic or cultural background?

Aboriginal/First Nation

Arab

Black

Chinese

Filipino

Japanese

Korean

Latin American

South Asian (e.g. Indian, Pakistani, Sri Lankan)

Southeast Asian (e.g. Vietnamese, Cambodian, Malaysian)

West Asian (e.g. Iranian, Afghan)

White

Other, please specify:

Prefer not to say 
Are you an international student?
Yes
No
Prefer not to say

Is English your first language?
Yes
No
Prefer not to say

Where do you currently live?

Campus residency hall, please specify which one:

Fraternity or sorority house

Other university housing, please specify:

\section{Parent's or guardian's home}

In a house/apartment by myself

In a house/apartment with room mates

Other off-campus housing, please specify:

\section{Prefer not to say}


What is your current year in school?

1st year undergrad

2nd year undergrad

3rd year undergrad

4th year undergrad

Higher than 4th year undergrad

Graduate or professional

Not seeking a degree

Other, please specify:

Prefer not to say

What is your field of study?

\section{End of Block: Main Survey}

